\title{
Dynamical connection between tropospheric blockings and stratospheric polar vortex
}

\author{
J. M. Castanheira ${ }^{1}$ and D. Barriopedro ${ }^{2}$ \\ Received 4 May 2010; revised 3 June 2010; accepted 8 June 2010; published 14 July 2010.
}

[1] The dynamical connection between Northern Hemisphere blocking events and the variability of the stratospheric polar vortex strength is studied. The analysis is based on the composite time evolution of the energy of baroclinic planetary waves during regional blocking occurrence. During Euro-Atlantic blocking events, an in phase forcing of stationary zonal wavenumber 1 occurs. The enhanced wave amplitude is associated with a stratospheric polar vortex deceleration, which may result, at times, in Sudden Stratospheric Warming (SSW) events of displacement type. Pacific blocking composites reveal an in phase forcing of stationary zonal wavenumber 2 . In most cases, the amplification of the wavenumber 2 does not reduce the vortex strength, being even accompanied by a mean vortex acceleration. However, if the amplification of wavenumber 2 is preceded by an amplification of wavenumber 1 , the initial vortex deceleration forced by wavenumber 1 may be continued by wavenumber 2 , and a SSW event of splitting type may occur. Citation: Castanheira, J. M., and D. Barriopedro (2010), Dynamical connection between tropospheric blockings and stratospheric polar vortex, Geophys. Res. Lett., 37, L13809, doi:10.1029/2010GL043819.

\section{Introduction}

[2] SSW events represent extreme weakening or even a reversing of the polar night cyclonic vortex. Large stratospheric anomalies progress downwards and are associated with regime shifts of surface circulation that may persist up to two months [Thompson et al., 2002]. Blocking events are quasi-stationary large departures from the mean zonal flow that persist longer than the typical synoptic time scales, impacting significantly on mid-latitude regional climates. Hence, both phenomena are of interest for extended weather prediction, climate variability and climate change.

[3] The winter stratospheric dynamics is largely forced by planetary waves propagating upwards from the troposphere [e.g., Andrews et al., 1987], and strong stratospheric circulation anomalies appear associated with strong anomalies in the upward heat flux [e.g., Polvani and Waugh, 2004; Charlton and Polvani, 2007]. In this context, large-scale long-lasting tropospheric disturbances, such as blocking events, and extreme stratospheric events such as SSWs have long been believed to be linked [e.g., Labitzke, 1965; Quiroz, 1986]. This issue has been revisited in three recent

\footnotetext{
${ }^{1}$ CESAM, Department of Physics, University of Aveiro, Aveiro, Portugal.

${ }^{2}$ CGUL, IDL, Faculty of Sciences, University of Lisbon, Lisbon, Portugal.

Copyright 2010 by the American Geophysical Union. 0094-8276/10/2010GL043819
}

studies by Taguchi [2008], Martius et al. [2009] and Woollings et al. [2010] (hereafter T08, M09 and W10, respectively). The results of T08 suggest that there is no lagged or simultaneous connection between tropospheric blockings and SSWs, whereas the results of M09 and W10 add evidence on the forcing of SSWs events by preexisting tropospheric blockings. Two conflicting results between the studies of M09 and W10 are the geographical location of blockings preceding SSWs of splitting type, and the role of planetary wavenumbers 1 and 2 on the forcing of SSWs. M09 suggest that "vortex displacement events are nearly always preceded by blocking over the Atlantic basin only, whereas vortex splitting events are preceded by blocking events occurring in the Pacific basin or in both basins contemporaneously". On the other hand, W10 claim that both vortex disturbances are mainly forced by Euro-Atlantic blockings. Moreover, M09 show that wavenumber 1 and 2 anomalies precede SSWs of displacement and split type, respectively, whereas W10 suggest that wavenumber 2 anomalies are the most evident forcing signal in the two types of SSWs.

[4] Our study tries to clarify the conflicting results of the aforementioned studies, shedding light on the connection between tropospheric blocking events and stratospheric polar vortex variability.

\section{Methods and Data}

[5] The analysis is based on daily fields obtained from the NCEP-NCAR reanalysis [Kalnay et al., 1996] and the ERA-40 reanalysis [Uppala et al., 2005], for the active Northern Hemisphere (NH) stratosphere-troposphere interaction 'season', namely November to March (NDJFM), in the common reanalysis period (1958-2001). Blocking events were identified in the ERA-40 reanalysis using a potential vorticity (PV)-based algorithm similar to that used by Schwierz et al. [2004] (details of the adaptation of Schwierz et al.'s method are in the auxiliary material), and in the NCEP-NCAR reanalysis using a recent methodology developed by Barriopedro et al. [2010] based on 500-hPa geopotential heights. ${ }^{3}$ Both indices consider blocking events as large-scale two dimensional (2-D) patterns with life-time exceeding 5 days. The use of two blocking indexes and two datasets allows for a test of results' sensitivity to both the blocking detection algorithm and the analyzed data.

[6] Because the coupled troposphere-stratosphere dynamics associated with SSWs must be similar with that of other vortex disturbances not catalogued as SSWs, we follow here the approach of W10 and analyze the relationship between blocking events and the variability of the vortex strength in

\footnotetext{
${ }^{3}$ Auxiliary materials are available in the HTML. doi:10.1029/ 2010GL043819.
} 
the full dataset. The identified associations were then checked in the smaller sample of SSW events. The vortex strength is represented by the 50-hPa NH annular mode (NAM) index of Baldwin and Dunkerton [2001], and SSW events were stratified into displacement and splitting events according to the Table 1 of Charlton and Polvani [2007].

[7] The NCEP-NCAR horizontal wind and geopotential fields were projected onto a normal mode basis of 3-D functions already available from previous works of the leading author [e.g., Castanheira et al., 2009]. The projections allow the decomposition of the atmospheric circulation onto barotropic and baroclinic vertical structures, and onto zonal and meridional wavenumber spectra of divergent (gravitic) and rotational (Rossby) waves. Because the interest is on vertically propagating waves, the analysis will focus on the baroclinic waves only. Wave variability is measured by the anomalies of the wave energy, which is proportional to the squared wave amplitude. Daily anomalies were computed by subtracting the seasonal cycle from the energy of each wave. The seasonal cycle was estimated by computing the multiannual mean for each calendar day and then smoothing with a 31-day moving average.

[8] Associations between the vortex strength, the energy of baroclinic Rossby waves and the occurrence of blocking events were investigated by performing composites of the energy anomalies and the 50-hPa NAM with respect to the onset dates of the blocking events or the central dates of SSWs. Following the analyses of M09 and W10, the composites were performed grouping the blocking events on a regional basis, according to their mean geographical positions. Four blocking sectors were considered: Euro-Atlantic $\left[40^{\circ} \mathrm{W}-50^{\circ} \mathrm{E}\right]$, Asia $\left[50^{\circ} \mathrm{E}-140^{\circ} \mathrm{E}\right]$, Pacific $\left[140^{\circ} \mathrm{E}-230^{\circ} \mathrm{E}\right]$ and North America $\left[130^{\circ} \mathrm{W}-40^{\circ} \mathrm{W}\right]$. The choice of sectors was suggested by the zonal patterns of the climatological wavenumber 1 and 2 waves (see supplementary material). The results are not qualitatively different if the sectors are rotated by 10 to $20^{\circ} \mathrm{N}$, but the chosen partition is the one that provides the strongest associations for the PV-blocking index. The blocking index of Barriopedro et al. [2010] provides qualitatively similar but less clear results, and in the following the composites will be shown for the PV index only. These composites imply crossing data derived from the two reanalysis datasets. Although differences in reanalysis have small influence in blocking detection, the cross of data adds more confidence to the resulting statistics. The statistical significance of the composites was determined by a Monte Carlo approach using 1000 (10000) trials in which the years of the blockings (SSWs) are randomly chosen between the 44 years of the dataset, keeping the days and months intact in order to take into account possible seasonal effects.

\section{Results}

[9] Figure 1 shows the composites of the vortex strength and the energy anomalies of baroclinic Rossby waves for the four sectors defined above. Overall, the mean vortex strength responds to changes in the energy of wavenumber 1 , with vortex deceleration (acceleration) being associated with an increase (decrease) in the wavenumber 1 energy. Regional differences in the relationship between vortex strength and blocking are also evident. In agreement with the results of $\mathrm{W} 10$, there is a preconditioning of the extratropical atmo- spheric circulation characterized by an anomalously strong mean vortex preceding Euro-Atlantic blockings. Similar behaviour is also observed in the Asian sector, but in this case the vortex deceleration occurs before the blocking onset. The occurrence of significant positive energy anomalies in the wavenumber 1 during the vortex deceleration suggests a coupled tropospheric-stratosphere process anticipating the onset of Asian blocks. This is in agreement with Wang et al. [2009], who found that Ural blockings are influenced by wave activity propagating from the Atlantic basin. Moreover, the deceleration of strong vortex states may provide some skill to the forecasting of Asian blockings. In fact, taking the 51 deceleration events from strong vortex states analyzed by Castanheira et al. [2009], 24 (9) Asian blocking onsets were identified in the 20 days after (before) the beginning of the vortex deceleration, the difference being statistically significant at the $99 \%$ confidence level $(p=0.005$, two-sided test).

[10] Pacific blockings are associated with positive anomalies in the energy of wavenumber 2 and negative anomalies in the energy of wavenumber 1, and, on average, they precede a vortex strengthening (Figure 1). The mean vortex acceleration, even with the amplification of wavenumber 2, must result from the dominant effect of the diminished amplitude of wavenumber 1 , which propagates more effectively into the vortex [Charney and Drazin, 1961].

[11] Euro-Atlantic and Pacific blockings present opposite effects in the energies of wavenumbers 1 and 2 . Therefore, the anomalies are expected to be larger if the composites are performed for blocking events in one sector that are not coincident with blocking events in the other. An EuroAtlantic (Pacific) blocking is considered a not-coincident event if its onset is separated by at least five days from the onset of any block in the Pacific (Euro-Atlantic) sector. The larger composite anomalies, shown in Figure 1 (bottom), corroborate the existence of a destructive interference between Euro-Atlantic and Pacific blocking wave forcings mainly for wavenumber 1 .

[12] Figure 2 shows the composites of wavenumber 1 and 2 in the physical space, allowing for a simple interpretation of the above results. Euro-Atlantic blockings interfere constructively with the climatological stationary wavenumber 1 (see also supplementary material), thus enhancing its amplitude. On the other hand, Pacific blockings interfere destructively with the climatological stationary wavenumber 1 but constructively with climatological stationary wavenumber 2. Thus, Pacific blocking forcings of wavenumbers 1 and 2 are opposite.

[13] SSW events result from dynamical forcings similar to those producing vortex decelerations in general. Figure 3 shows the composites of the vortex strength and the energy anomalies of baroclinic Rossby waves for SSWs of splitting and displacement type. SSWs of displacement type are preceded by an extended period of strong positive energy anomalies in the wavenumber 1 that is followed by strong negative anomalies of the vortex strength. SSWs of splitting type show different dynamics. In the period 25 to 15 days before the SSW central date, strong positive energy anomalies in the wavenumber 1 are observed. They are accompanied by a vortex weakening and followed by strong positive energy anomalies of wavenumber 2 in the 11 days before the SSW central date. A possible interpretation of this result is that after the previous deceleration by wavenumber 1 , 


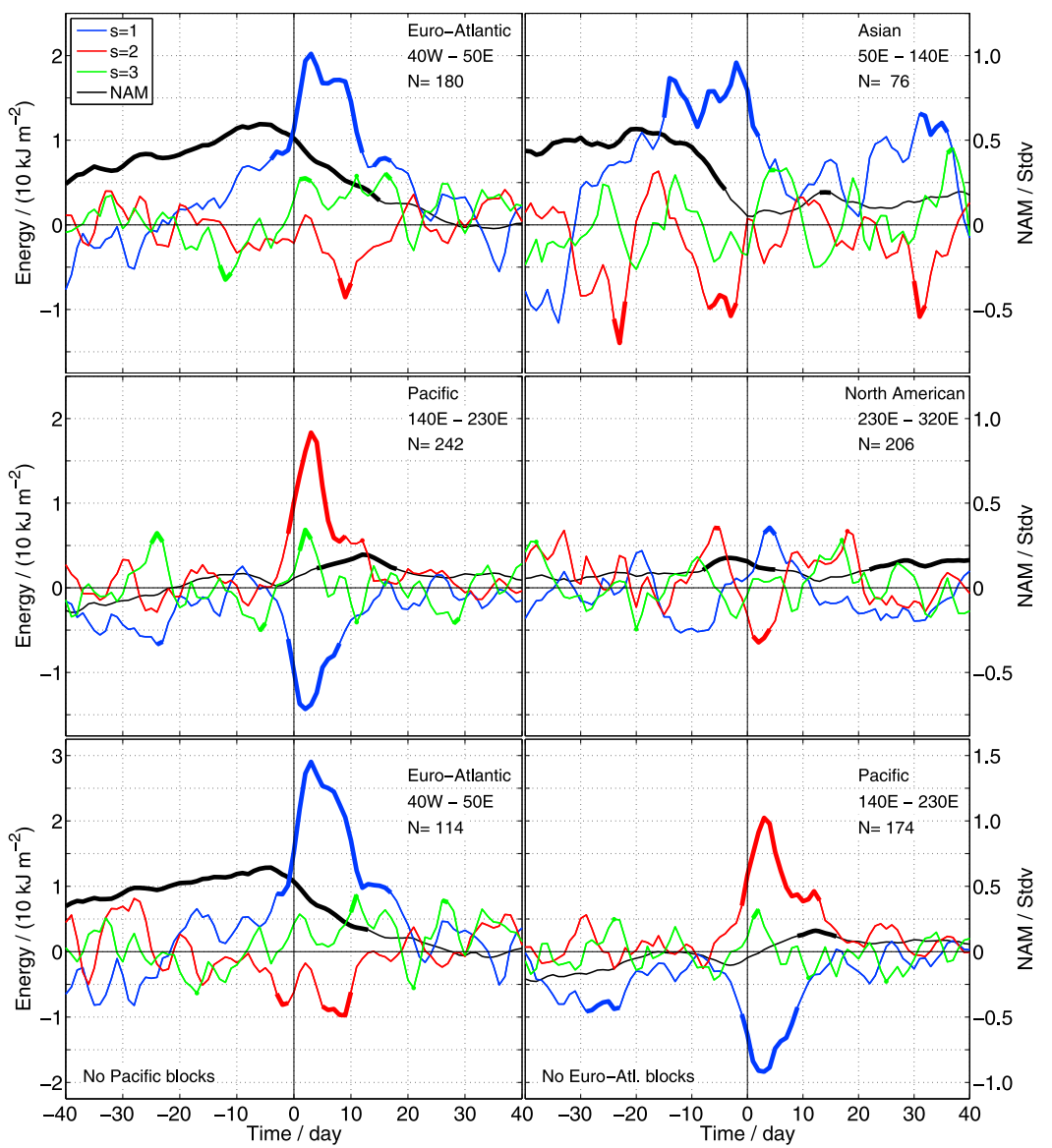

Figure 1. Composites of daily 50-hPa NAM (black lines) and energy anomalies of baroclinic Rossby waves with zonal wavenumbers $s=1,2$ and 3 (colour lines) for Euro-Atlantic, Asian, Pacific and North American blocking events. Sector's longitudes and the number, $N$, of events are indicated in each plot. Bottom panels show the composites when no coincident blocking occurs in the opposite sector. (Note the scale change in the vertical-axis.) Day zero refers to the blocking onsets. Thick line segments indicate anomalies significantly different from zero at the $95 \%$ confidence level $(p=0.025$, two-sided test).

the vortex is less refractive for wavenumber 2 , which then can further decelerate the vortex.

[14] M09 and W10 reported regional connections between blocking and SSW events. Figures 1 and 3 together also suggest the existence of statistical regional connections. It is expected that more Euro-Atlantic blocking events occur before than after SSWs of displacement type. On the other hand, more blocking events are expected to occur both in the Euro-Atlantic and in the Pacific sectors before than after SSWs of splitting type. Table 1 shows the number, $N$, of blocking events observed in the 20 days periods before and after SSW events for each sector. The differences between the numbers of blocking events are in line with the expectation, being statistically significant at the $95 \%$ confidence level. Interestingly, the number of Pacific blocks before vortex displacements is also anomalously small, in agreement with the proposed destructive interference between Pacific and Euro-Atlantic blocks for the forcing of wavenumber 1. Although Figures 1 and 3 and Table 1 suggest that the direction of influence is upwards, it must be noted that the frequency of Euro-Atlantic blocking is anomalously low after the SSWs. This is in line with the above mentioned preconditioning of the atmospheric circulation characterized by an anomalously strong mean vortex preceding EuroAtlantic and Asian blockings.

\section{Concluding Discussion}

[15] Our results confirm the findings of M09 and W10 which point for a regional-dependent connection between blocking and stratospheric variability. Blocks occurring at different geographical regions present different associations with the stratospheric polar vortex strength, and this may explain the lack of such association when blocks are analyzed together as done by T08. Furthermore, our results shed more light on the connection between blocking events and stratospheric polar vortex variability, contributing to clarify the conflicting results in the previous studies.

[16] The most important difference between our results and those of W10 concerns the role played by the wavenumbers 1 and 2 in the forcing of stratospheric variability. Herein, the strength of the atmospheric polar vortex responds mainly to the wavenumber 1, which is amplified by the occurrence of Euro-Atlantic blockings, whereas in W10, the main driver of stratospheric variability associated with European blocking appears to be the wavenumber 2 . 

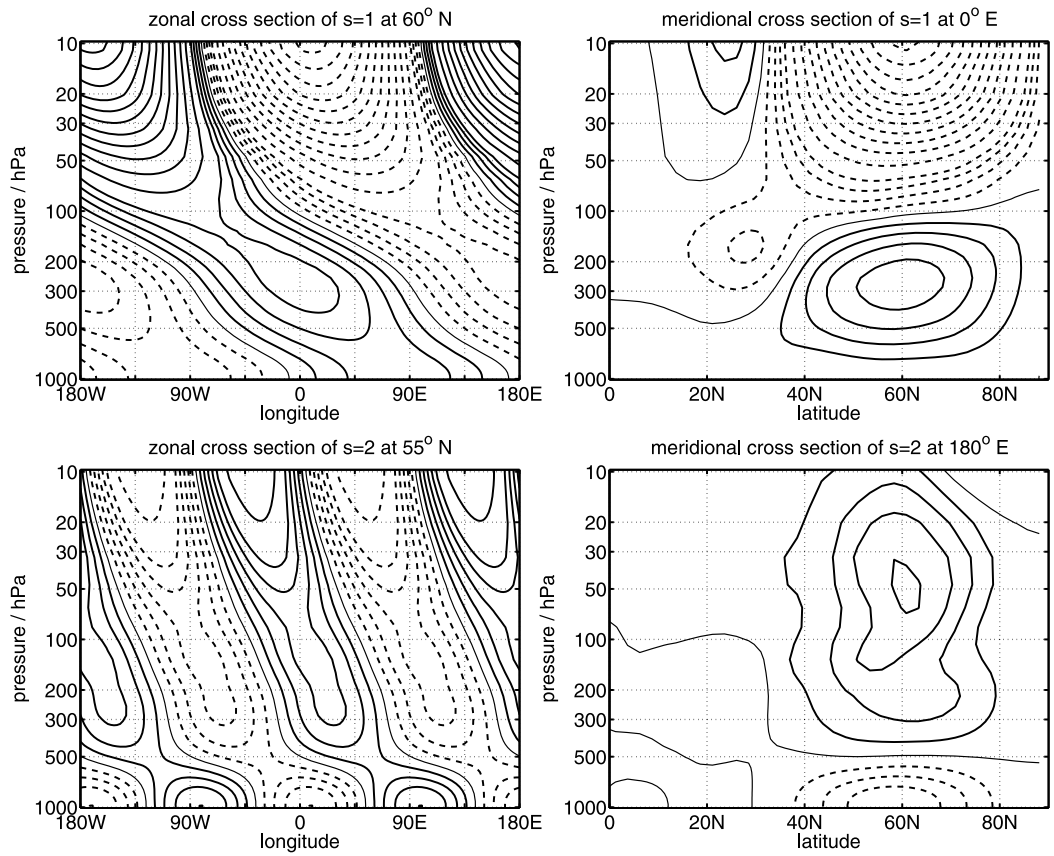

Figure 2. Composites of baroclinic Rossby waves with zonal wavenumbers (top) 1 and (bottom) 2 for 5 days periods centered at day 3 after blocking onsets. Top (bottom) panels show composites for Euro-Atlantic (Pacific) blocking events not coincident with blocking events in the Pacific (Euro-Atlantic) sector. Dashed lines indicate negative values. Contour intervals are 25 (20) gpm, between -100 and 100, and 50 (40) gpm for large absolute values in the top (bottom) panels.

If Euro-Atlantic blocking has some impact on the wavenumber 2, our results point for small negative energy anomalies. In what concerns Pacific blocking, both studies suggest an amplification of wavenumber 2. Another common result is that the vortex is strong before the occurrence of Euro-Atlantic blockings.

[17] In addition to the differences caused by the blocking definition (see W10 for a deeper discussion), the measures of wave variability are also different in our study and that of W10. We used the wave energy whereas W10 used the quasi-geostrophic wave activity. Besides the methodological aspects, there is also an approach in the calculations of W10 that may contribute for the differences found in the roles played by wavenumbers 1 and 2: they used a climatological quasi-geostrophic PV gradient $\left(q_{y}\right)$ in the denominator of the expression for the wave activity. Time variations of $q_{y}$ are crucial to explain changes in strato- spheric vortex refractivity to planetary waves. For instance, $q_{y}$ may be appreciably different during strong and weak vortex states, as those preceding Euro-Atlantic and Pacific blocking, respectively (see Figure 1, bottom).

[18] The analysis of SSW composites confirms the different roles played by wavenumbers 1 and 2 . In agreement with M09, SSWs of displacement type are preceded by an amplification of wavenumber 1 (Figure 3) and EuroAtlantic blocking episodes occurs more before than after displacement SSWs (Table 1). Vortex splittings are preceded by an amplification of wavenumber 1 in the period 25-15 days before the SSWs. It seems that the amplification of wavenumber 1 forces a previous vortex deceleration, then allowing for the wavenumber 2 to cause further vortex deceleration in the 11 days before the SSW. It must be remarked that M09 suggest a simultaneous (in the 10 days before SSWs) amplification of wavenumbers 1 and 2,

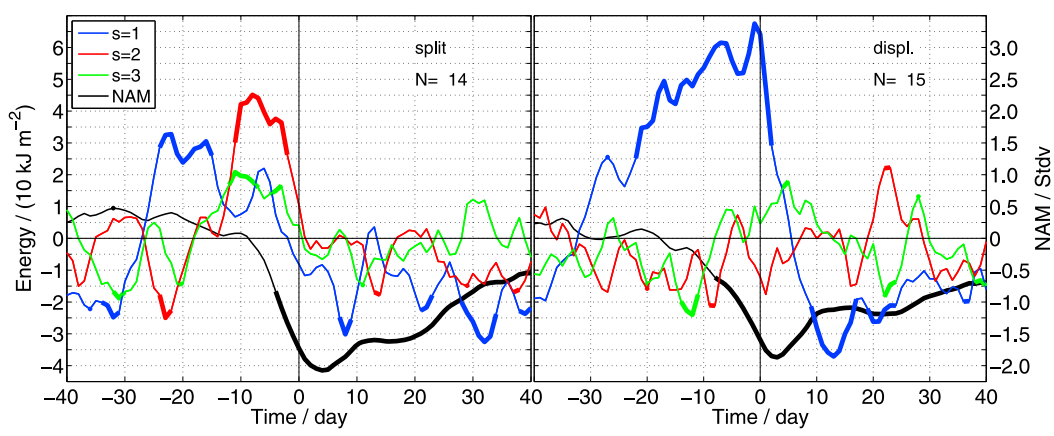

Figure 3. As in Figure 1 but for SSWs of (left) splitting and (right) displacement type. Day zero refers to the central dates of SSWs. Thick portions of the lines indicate that the mean energy anomalies are significantly different from zero at the $95 \%$ confidence level $(p=0.05$, one sided test). 
Table 1. Total Number of Blocking Events Observed in the 20 Days Periods Before and After SSWs Events for Each Sector ${ }^{\mathrm{a}}$

\begin{tabular}{lccc}
\hline \multicolumn{1}{c}{ Sector } & $\begin{array}{c}N \text { Blockings } \\
\text { Before SSWs }\end{array}$ & $\begin{array}{c}N \text { Blockings } \\
\text { After SSWs }\end{array}$ & Difference \\
\hline Euro-Atlantic & \multicolumn{2}{c}{ Displacement } & $\mathbf{8}$ \\
Asian & 12 & 5 & $\mathbf{8}$ \\
Pacific & 4 & 11 & -1 \\
North American & $\mathbf{8}$ & 9 & -3 \\
& 15 & & 6 \\
Euro-Atlantic & & $\mathbf{1}$ & \\
Asian & 7 & 2 & $\mathbf{6}$ \\
Pacific & 3 & 13 & $\mathbf{8}$ \\
North American & $\mathbf{2 1}$ & 13 & -3 \\
\hline
\end{tabular}

${ }^{\mathrm{a}} N$, total number. Bolded values are statistically significant at the $95 \%$ confidence level ( $\mathrm{p}=0.05$, one-sided test). Only 14 SSWs of displacement type were considered here, because one SSW included in Figure 3 falls out of the period of blocking data.

whereas our results suggest a time delay between the amplifications of wavenumber 1 and 2. In fact, from Figure 2 of M09 one cannot conclude immediately for an amplification of wavenumber 1 and 2 before SSW splittings. The observed increasing amplitude with altitude does not necessarily mean an amplification of the wave because it was not weighted by the square root of density. However, from the comparison of the two plots of their Figure 2, it is clear that the wavenumber 1 (2) has larger amplitudes before displacement (splitting) SSWs than before splitting (displacement) SSWs, and this agrees with the results found herein. Moreover, Table 1 reveals higher frequency of both Euro-Atlantic and Pacific blockings in the 20 days period before than after splitting SSWs, also in agreement with M09.

[19] Finally, although most of the results suggest an upward blocking influence, a preconditioning of the atmospheric circulation characterized by a strong stratospheric vortex before the occurrence of Euro-Atlantic and Asian blockings is also apparent.

\section{References}

Andrews, D. G., J. R. Holton, and C. B. Leovy (1987), Middle Atmosphere Dynamics, 489 pp., Academic, San Diego, Calif.
Baldwin, M. P., and T. J. Dunkerton (2001), Stratospheric harbingers of anomalous weather regimes, Science, 294, 581-584.

Barriopedro, D., R. García-Herrera, and R. M. Trigo (2010), Application of blocking diagnosis methods to general circulation models. Part I: A novel detection scheme, Clim. Dyn., doi:10.1007/s00382-010-0767-5.

Castanheira, J. M., M. L. R. Liberato, L. de la Torre, H.-F. Graf, and C. C. DaCamara (2009), Baroclinic Rossby wave forcing and barotropic Rossby wave response to stratospheric vortex variability, J. Atmos. Sci., 66, 902-914.

Charlton, A. J., and L. M. Polvani (2007), A new look at stratospheric sudden warmings. Part I. Climatology and modelling benchmarks, J. Clim., 20, 449-469.

Charney, J. G., and P. G. Drazin (1961), Propagation of planetary-scale disturbances from the lower into the upper atmosphere, J. Geophys. Res., $66,83-109$.

Kalnay, E., et al. (1996), The NCEP/NCAR 40-year reanalysis project, Bull. Am. Meteorol. Soc., 77, 437-471.

Labitzke, K. (1965), On the mutual relation between stratosphere and troposphere during periods of stratospheric warmings in winter, J. Appl. Meteorol., 4, 91-99.

Martius, O., L. M. Polvani, and H. C. Davies (2009), Blocking precursors to stratospheric sudden warming events, Geophys. Res. Lett., 36, L14806, doi:10.1029/2009GL038776.

Polvani, L. M., and D. W. Waugh (2004), Upward wave activity flux as a precursor to extreme stratospheric events and subsequent anomalous surface weather regimes, J. Clim., 17, 3548-3554.

Quiroz, R. S. (1986), The association of stratospheric warmings with tropospheric blocking, J. Geophys. Res., 91, 5277-5285.

Schwierz, C., M. Croci-Maspoli, and H. C. Davies (2004), Perspicacious indicators of atmospheric blocking, Geophys. Res. Lett., 31, L06125, doi:10.1029/2003GL019341.

Taguchi, M. (2008), Is there a statistical connection between stratospheric sudden warming and tropospheric blocking events?, J. Atmos. Sci., 65, 1442-1454.

Thompson, D. W. J., M. P. Baldwin, and J. M. Wallace (2002), Stratospheric connection to Northern Hemisphere wintertime weather: Implications for predictions, J. Clim., 15, 1421-1428.

Uppala, M., et al. (2005), The ERA-40 reanalysis, Q. J. R. Meteorol. Soc. 131, 2961-3012.

Wang, L., W. Chen, W. Zhou, J. C. L. Chan, D. Barriopedro, and R. Huang (2009), Effect of the climate shift around mid 1970's on the relationship between wintertime Ural blocking circulation and East Asian climate, Int. J. Climatol., 30, 153-158, doi:10.1002/joc.1876.

Woollings, T., A. Charlton-Perez, S. Ineson, A. G. Marshall, and G. Masato (2010), Associations between stratospheric variability and tropospheric blocking, J. Geophys. Res., 115, D06108, doi:10.1029/2009JD012742.

D. Barriopedro, CGUL, IDL, Faculdade de Ciências, Universidade de Lisboa, Campo Grande, Ed. C-8, P-1749-016 Lisbon, Portugal. (dbarriopedro@fc.ul.pt)

J. M. Castanheira, CESAM, Departamento de Física, Universidade de Aveiro, Campus de Santiago, P-3810-193 Aveiro, Portugal. (jcast@ua.pt) 